\title{
Efficacy and Safety of Complete Endoscopic Resection of Colorectal Neoplasia Using a Stepwise Endoscopic Protocol with SOUTEN, a Novel Multifunctional Snare
}

\author{
Shinji Yoshii ${ }^{1}$, Marina Kubo ${ }^{1}$, Mio Matsumoto ${ }^{2}$, Takefumi Kikuchi ${ }^{3}$ and Yasunari Takakuwa ${ }^{4}$ \\ ${ }^{1}$ Department of Gastroenterology, Sapporo Medical Center NTT EC, Sapporo, ${ }^{2}$ Department of Gastroenterology, Hokkaido Medical Center, \\ Sapporo, ${ }^{3}$ Division of Gastroenterology, Department of Internal Medicine, Sapporo Shirakabadai Hospital, Sapporo, ${ }^{4}$ Department of \\ Pathology, Sapporo Medical Center NTT EC, Sapporo, Japan
}

Background/Aims: A multifunctional snare SOUTEN has a sharp tip at the top of the snare loop that enables incision of the mucosa, dissection of the submucosal layer, and snaring of lesion. This study assessed the efficacy and safety of complete endoscopic resection of colorectal neoplasia using SOUTEN.

Methods: We analyzed the rates of gross en bloc resection and complete resections of 108 consecutive tumors from 69 patients resected by precutting endoscopic mucosal resection (precutting), hybrid endoscopic submucosal dissection (hybrid), or conventional endoscopic submucosal dissection (conventional) using SOUTEN.

Results: Out of the 108 tumors, 50 were resected by precutting, 27 were resected by hybrid after attempting precutting, and the remaining 31 were resected by conventional after attempting precutting and hybrid resections. The median tumor sizes were $14.5 \mathrm{~mm}$ for precutting, $16.4 \mathrm{~mm}$ for hybrid, and $21.1 \mathrm{~mm}$ for conventional. The success rate of gross en bloc resection and histological complete resection were $100 \%$ and $94.0 \%$ for precutting, $96.4 \%$ and $96.4 \%$ for hybrid, and $100 \%$ and $100 \%$ for conventional method, respectively. No procedure-related complication occurred.

Conclusions: By using SOUTEN, precutting and hybrid were successfully performed on 10-30 mm tumors with a shorter procedure time than conventional without major complications. Clin Endosc 2020;53:206-212

Key Words: Hybrid endoscopic submucosal dissection; Multifunctional snare; Precutting endoscopic mucosal resection; SOUTEN

\section{INTRODUCTION}

Conventional endoscopic mucosal resection (EMR) is a common technique for resecting colorectal neoplasia. A lesion less than $20 \mathrm{~mm}$ can be resected safely en bloc according to a Japanese guideline ${ }^{1}$ and a previous publication. ${ }^{2}$ This size limit is mainly because of technical difficulties as increase in size

Received: June 7, 2019 Revised: September 30, 2019

Accepted: December 12, 2019

Correspondence: Shinji Yoshii

Department of Gastroenterology, Sapporo Medical Center, NTT EC, S1 W15, Chuo-ku, Sapporo 060-0061, Japan

Tel: +81-11-623-7520, Fax: +81-11-11-623-7527, E-mail: shinji-yoshii@umin.ac.jp ORCID: https://orcid.org/0000-0002-5010-8357

(c) This is an Open Access article distributed under the terms of the Creative Commons Attribution Non-Commercial License (http://creativecommons.org/ licenses/by-nc/3.0) which permits unrestricted non-commercial use, distribution, and reproduction in any medium, provided the original work is properly cited. of a tumor results in increases in the chance of piecemeal resection. ${ }^{3-5}$ En bloc resection enables accurate histological diagnosis. Moreover, a meta-analysis ${ }^{6}$ showed that the recurrence risk of en bloc resection was reduced significantly compared to that of piecemeal resection ( $3 \%$ vs. $20 \%, p<0.0001)$. However, even when a lesion is smaller than $20 \mathrm{~mm}$, sometimes it may be difficult to resect using the conventional EMR because of its location or the existence of non-lifting signs. CARE $s t u d y^{2}$ reported that the rate of incomplete polyp resection was relatively high even in cases with lesions less than $20 \mathrm{~mm}$ (10-14 $\mathrm{mm}$ [13.4\%] and 15-20 $\mathrm{mm}$ [23.3\%] and in sessile serrated adenomas/polyps (SSA/Ps) [31.0\%]). To solve these problems, EMR with circumferential incision, including precutting EMR and hybrid endoscopic submucosal dissection (ESD), has been developed. ${ }^{7-9}$

A cost-effective, multifunctional snare (SOUTEN; Kaneka 
Medix, Tokyo, Japan) has a knob-shaped tip at the top of the loop (Fig. 1A-C). ${ }^{10}$ This tip prevents the needle-knife from slipping during circumferential incision and submucosal dissection. The loop functions as a snare when it expands. As this multifunctional snare has two different functions, cutting the mucosa and snaring the target lesion, all processes can be completed using just one device, resulting in an efficient and shorter endoscopic procedure. Further, this multifunctional snare is advantageous in that the endoscopist can change the procedure based on the lesion's location and visibility without the need for additional equipment. In this study, we assessed the safety and efficacy of performing our stepwise endoscopic protocol with this multifunctional snare.

\section{PATIENTS AND METHODS}

\section{Ethical statements}

Written informed consent to participate in this study was obtained from all the patients who underwent colonoscopy or any form of treatment. This retrospective study was approved by the institutional review board of Sapporo Medical Center, NTT EC (study approval number: 17-066) and was conducted in accordance with the Declaration of Helsinki.

\section{Patient selection}

We retrospectively analyzed the data of patients who underwent endoscopic treatment for neoplastic lesions by SOUTEN snare in Sapporo Medical Center, NTT EC from March 2017 to July 2018. A series of 108 lesions technically unsuitable for conventional EMR from 69 patients were selected for analysis.

\section{Definitions of procedures}

We followed the Japan Gastroenterological Endoscopy Society (JGES) guideline $e^{1}$ to define the procedures. A technique in which snaring is performed without dissecting the submucosal layer after incising the circumference of the lesion is defined as precutting EMR. A technique in which the submucosal layer is dissected and snaring is performed after ESD procedure is defined as hybrid ESD. Conventional EMR is defined as a snaring technique without incision or dissection of the submucosal layer. ${ }^{11}$

\section{Indications for precutting EMR and hybrid ESD}

The indication of conventional EMR was colorectal lesions that were considered difficult to be removed en bloc with polypectomy. ${ }^{11}$ In this study, we included the lesions that are technically unsuitable for conventional EMR because of its size, shape, especially a flat shape, and its association with colonic folds. We excluded pedunculated tumors.

\section{Endoscopic procedures using SOUTEN}

After a submucosal injection of hyaluronic acid solution to elevate the lesion, we cut the mucosa circumferentially around the lesion, and we tried precutting EMR first. When en block removal of the tumor was difficult by snaring, we continued dissection of the submucosal layer beneath the lesion to enable hybrid ESD. When either precutting EMR or hybrid ESD was difficult to perform, the conventional ESD technique was used to remove the tumor. We prioritized patients' safety and en block resection of tumors to ensure precise pathological diagnosis for a better prognosis.

During the endoscopic procedure, the endoscopists usually consider many factors to determine the best approach to remove the tumor safely and completely. Among these
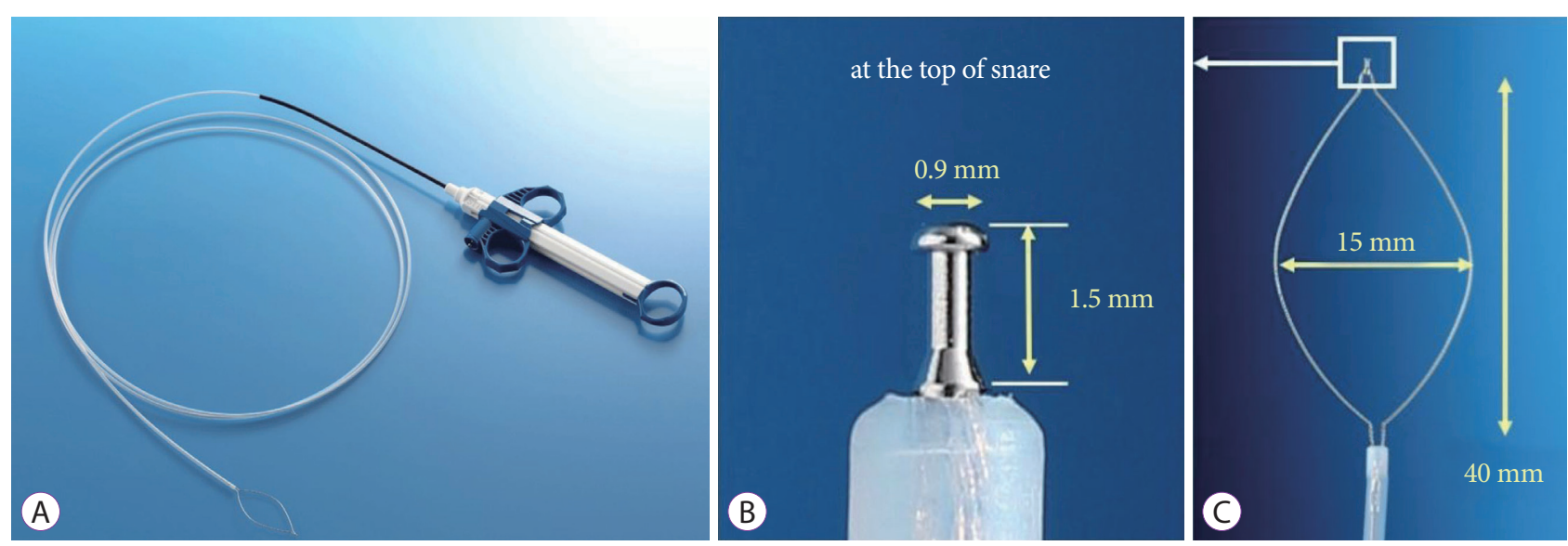

Fig. 1. Image of SOUTEN (Kaneka Medix, Tokyo, Japan) snare. (A) The multifunctional snare was designed to achieve precutting endoscopic mucosal resection and hybrid endoscopic submucosal dissection. (B) A 1.5-mm needle-knife with a knob-shaped tip was attached to the top of the loop. (C) The length of the loop was $40 \mathrm{~mm}$. 
procedures, we considered the following "difficult factors" to determine how difficult the target lesion was to be removed: depth of submucosal invasion, larger lesion size (more than $20 \mathrm{~mm}$ in diameter), sessile serrated histology, polyps location with respect to a colonic fold, polyps relationship with colonic flexure, and visibility of the lesion (tangentially). ${ }^{2,12}$ We did not perform prophylactic clipping to avoid postprocedural bleeding.

Supplementary video 1 shows an example of hybrid ESD using SOUTEN snare for a $30 \mathrm{~mm}$ granular laterally spreading tumor located in the lower rectum (Fig. 2A). In this video, a solution of hyaluronic acid (MucoUp; Boston Scientific Co., Ltd., Tokyo, Japan) with a slight amount of indigo carmine was injected to lift the submucosa (Fig. 2B). After submucosal injection, we incised the mucosa circumferentially $5 \mathrm{~mm}$ away from the tumor with the tip of the SOUTEN (Kaneka Medix) (Fig. 2C). At the endoscopist's discretion, trimmings were performed enough to prevent snare slippage (Fig. 2D), and the snare was inserted on the distal mucosal incision site and expanded gently towards the proximal side to fit the dissection plane, and the tumor was carefully snared (Fig. 2E-H).

\section{Pre-medication and procedure-related patient care}

All resection procedures were performed with the patient under conscious sedation: $2 \mathrm{mg}$ midazolam and $15 \mathrm{mg}$ pentazocine were administered intravenously, and sedation was maintained with intermittent injections of midazolam. Procedures were performed on all the patients by SY, who had colorectal ESD experience with more than 500 cases, and by two non-expert endoscopists supervised by SY, using a single-channel video endoscope with water jet function (PCFQ260JI, PCF-H290AI, or AZI; Olympus Optical Co., Ltd, Tokyo, Japan). A transparent attachment was used to make the lesion more visible. A VIO 300D electrosurgical unit (ERBE, Tubingen, Germany) was used. Mucosal incision and submucosal dissection with SOUTEN were conducted in endo cut I mode (effect 2, duration 2, interval 2) and swift coagulation mode (effect 3, $45 \mathrm{~W}$ ), respectively. Snare resection was conducted in endo cut I mode (effect 2, duration 2, and interval 2). Carbon dioxide insufflation was used to reduce patients' discomfort.

Antiplatelet agents were discontinued before the procedure according to JGES guidelines. ${ }^{1}$ Aspirin was administered to patients with a high cardiovascular risk. Antiplatelet agents resumed the next day of the procedure when there was no hematochezia or melena.

\section{Pathological evaluation}

The specimens were fixed in formalin and serially sectioned at $2 \mathrm{~mm}$ intervals to assess tumor involvement in the lateral and vertical margins. We assessed the histopathologic findings according to the Japanese Society for Cancer of the Colon and Rectum guidelines. ${ }^{5}$ Histopathologic findings were evaluated by a board-certified gastrointestinal pathologist in our hospital (YT). Pathological diagnoses were based on Vienna classification. $^{13}$
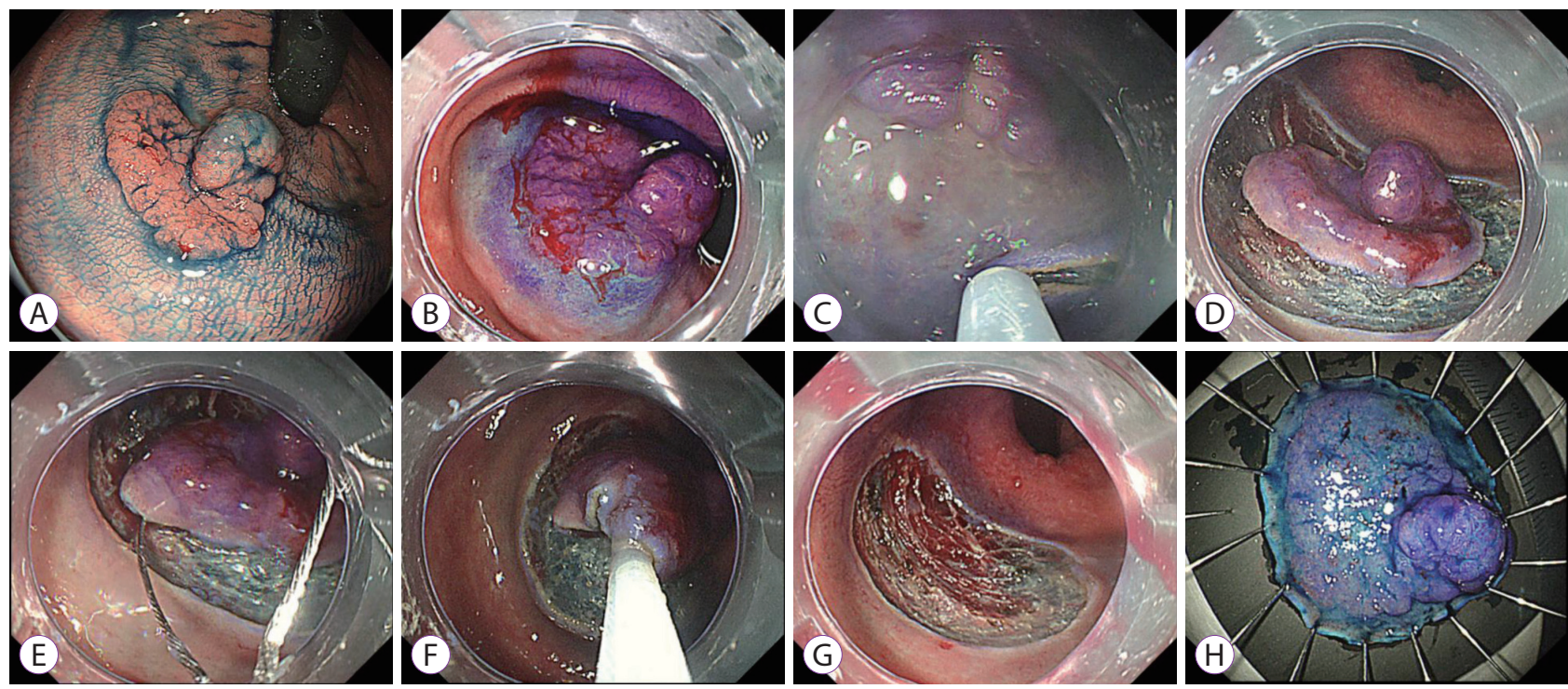

Fig. 2. (A) A 30-mm granular type laterally spreading tumor was located in the lower rectum. (B) Local injection of sodium hyaluronate solution. (C) Mucosal incision was performed using a needle-knife of the top of the snare. (D) Adequate trimming was performed. (E) The snare was placed on the dissection plane. (F) The tumor was tightly snared. (G) Post-precutting endoscopic mucosal resection. The lesion was completely resected. $(\mathrm{H})$ The resected specimen. En bloc resection was achieved. 


\section{Outcomes}

Procedure-related outcomes, including procedure time, snaring time, gross en bloc resection rate, histologic complete resection rate, and adverse events, were retrieved from patients' medical records retrospectively. Procedure-related adverse events included postprocedural bleeding and perforation.

\section{Statistical analysis}

All statistical analyses were performed with EZR (Saitama Medical Center, Jichi Medical University, Saitama, Japan), which is a graphical user interface for $\mathrm{R}$ (The R Foundation for Statistical Computing, Vienna, Austria). ${ }^{14}$ Kruskal-Wallis test and Bonferroni's modification of Mann-Whitney $U$ test were used for the analysis of lesion size, sample size, and procedure time.

\section{RESULTS}

Patients' characteristics and lesion size are summarized in Table 1 . We analyzed 108 lesions predominantly from women in their 60s. Out of the 108 tumors, 50 tumors were resected by precutting EMR, 27 tumors were resected by hybrid ESD after trying precutting EMR, and the remaining 31 tumors were resected by conventional ESD after trying precutting EMR and hybrid ESD. The median tumor sizes were $14.5 \mathrm{~mm}$ for precutting EMR, $16.4 \mathrm{~mm}$ for hybrid ESD, and $21.1 \mathrm{~mm}$ for conventional ESD. We found a statistically significant difference in lesion size between the three procedures; significantly larger lesions were resected with conventional ESD than with precutting EMR and hybrid ESD. The number of lesions measuring more than $20 \mathrm{~mm}$ in diameter was significantly higher in conventional ESD than in precutting EMR and hybrid ESD.
The location of lesions, and the size, morphology, and histopathology of resected materials are summarized in Table 2. There was no significant difference in the location, morphology, and association with colonic folds between the three procedures. There was also no statistical significance in visibility between the three procedures. However, more than half of the lesions (58.1\%) were tangentially visible with conventional ESD, whereas more than half of the lesions were vertically visible with precutting EMR and hybrid ESD.

Procedure time and outcome are summarized in Table 3. The average procedure times were 3.7 minutes in precutting EMR, 7.7 minutes in hybrid ESD, and 17 minutes in conventional ESD. There was no statistical significance in procedure time between precutting EMR and hybrid ESD, but procedure time was longer with conventional ESD than with precutting EMR or hybrid ESD. The success rates of gross en bloc resection and histological complete resection were $100 \%$ and 94.0\% for precutting EMR, 96.4\% and 96.4\% for hybrid ESD, and $100 \%$ and $100 \%$ for conventional ESD. There was no procedure-related complication such as perforation or bleeding. Out of the 108 lesions we analyzed, detailed analysis revealed that 91 lesions (83.4\%) were difficult lesions defined by the difficult factors. The multifunctional snare and our stepwise endoscopic protocol enabled us to perform safe endoscopic procedures for difficult lesions.

\section{DISCUSSION}

The multifunctional snare SOUTEN enables endoscopists to perform several different procedures without changing the device and incurring additional costs. As every lesion is located in different parts of the colon with different conditions, such as visibility of the lesion and the association with colonic folds, we challenged our stepwise endoscopic protocol

Table 1. Characteristics of the Patients and Lesions

\begin{tabular}{lccc}
\hline & Precutting EMR & Hybrid ESD & Conventional ESD \\
\hline Number of lesions & 50 & 27 & 31 \\
Sex $(\mathrm{M} / \mathrm{F})$ & $15 / 35$ & $9 / 18$ & $14 / 17$ \\
Age $(\mathrm{yr})($ mean \pm SD) & $61.3 \pm 12.1$ & $65.4 \pm 11.5$ & $63.7 \pm 14.0$ \\
Mean lesion size $(\mathrm{mm})($ mean $\pm \mathrm{SD})$ & $14.5 \pm 5.4$ & $16.4 \pm 6.3$ & $21.1 \pm 7.7^{\mathrm{a})}$ \\
Range of the lesion size (mm) & $8-28$ & $5-28$ & $8-39$ \\
Number of lesions & & & $19(17.6)$ \\
Less than $20 \mathrm{~mm}(\%)$ & $39(36.1)$ & $8(7.4)$ & $10(9.3)$ \\
More than $20 \mathrm{~mm}(\%)$ & $11(10.2)$ & $21(19.4)$ \\
\hline
\end{tabular}

EMR, endoscopic mucosal resection; ESD, endoscopic submucosal dissection; SD, standard deviation.

${ }^{\text {a) }}<<0.01$ comparing with precutting EMR. 
to achieve a shorter procedure time and higher en block resection rates. From the patients' perception, minimally invasive therapy reduces their burden and decreases procedure-related complications. In this study, we first tried precutting EMR; however, when the lesion was difficult to remove, additional submucosal dissection was performed. Next, we tried hybrid

Table 2. Summary of Specimen Size, Location, Morphology, and Histopathology

\begin{tabular}{|c|c|c|c|}
\hline & Precutting EMR & Hybrid ESD & Conventional ESD \\
\hline Number of lesions & 50 & 27 & 31 \\
\hline Mean specimen size $(\mathrm{mm} \pm \mathrm{SD})$ & $18.8 \pm 5.4$ & $21.4 \pm 6.3$ & $25.7 \pm 7.7^{\mathrm{a})}$ \\
\hline Range of the specimen size (mm) & $10-35$ & $10-32$ & $12-44$ \\
\hline \multicolumn{4}{|l|}{ Location of lesions } \\
\hline Right/left/rectum & $36 / 12 / 2$ & $18 / 7 / 2$ & $23 / 3 / 5$ \\
\hline \multicolumn{4}{|l|}{ Colonic folds } \\
\hline Between folds (\%) & $37(74.0)$ & $17(62.9)$ & $22(71.0)$ \\
\hline Across a fold (\%) & $13(26.0)$ & $10(37.0)$ & $9(29.0)$ \\
\hline \multicolumn{4}{|l|}{ Colonic flexure } \\
\hline In non-flexure (\%) & $44(88.0)$ & $23(85.2)$ & $25(80.6)$ \\
\hline In flexure (\%) & $6(12.0)$ & $4(14.8)$ & $6(19.4)$ \\
\hline \multicolumn{4}{|l|}{ Visibility } \\
\hline Vertically (\%) & $32(64.0)$ & $16(59.3)$ & $13(41.9)$ \\
\hline Tangentially (\%) & $18(36.0)$ & $11(40.7)$ & $18(58.1)$ \\
\hline \multicolumn{4}{|l|}{ Morphology } \\
\hline $0-\mathrm{I} / 0-\mathrm{II}$ & $9 / 41$ & $4 / 23$ & $12 / 19$ \\
\hline \multicolumn{4}{|l|}{ Histopathology } \\
\hline TA or TSA & 29 & 13 & 17 \\
\hline $\mathrm{SSA} / \mathrm{P}$ & 15 & 7 & 8 \\
\hline Tis & 1 & 4 & 3 \\
\hline $\mathrm{T} 1$ & 4 & 2 & 2 \\
\hline Hyperplastic polyp & 1 & 1 & 1 \\
\hline
\end{tabular}

The specimen size is the size of the whole resected specimen, whereas the lesion size is the size of the tumor in the resected specimen. EMR, endoscopic mucosal resection; ESD, endoscopic submucosal dissection; SD, standard deviation; SSA/P, sessile serrated adenoma/polyp; TA, tubular adenoma; TSA, traditional serrated adenoma.

${ }^{\text {a) }} P<0.01$ comparing with precutting EMR, $p<0.05$ comparing with hybrid ESD.

Table 3. Summary of the Endoscopic Procedure Time and Outcome

\begin{tabular}{lccc}
\hline & Precutting EMR & Hybrid ESD & Conventional ESD \\
\hline Number of samples & 50 & 27 & 31 \\
Procedure time (sec \pm SD) & $221.7 \pm 139.6$ & $461.0 \pm 466.2$ & $1139.1 \pm 755.2^{\mathrm{a})}$ \\
Snaring time (sec) & 52.1 & 58.4 & Not applicable \\
Gross en bloc resection (\%) & 100 & 96.4 & 100 \\
Histological complete resection (\%) & 94 & 96.4 & 100 \\
Adverse events & & & 0 \\
Bleeding & 0 & 0 & 0 \\
Perforation & 0 & 0 & 0 \\
\hline
\end{tabular}

EMR, endoscopic mucosal resection; ESD, endoscopic submucosal dissection; SD, standard deviation.

${ }^{\text {a) }} P<0.01$ comparing with both precutting EMR and hybrid ESD. 
ESD, and when the lesion was still difficult to resect, it was removed by conventional ESD involving full dissection of the submucosal layer.

As we described in the Results section, the procedure time was shorter in pre-cutting EMR and hybrid ESD than in conventional ESD. Pre-cutting EMR and hybrid ESD are relatively quicker than conventional ESD because the remaining submucosal layer is removed using a snare without the needle-knife. ${ }^{15,16}$ Pre-cutting EMR and hybrid ESD require a cutting device, such as an ESD knife, in addition to a snare, and it costs higher than conventional EMR. However, using this multifunctional snare SOUTEN resulted in no additional cost.

The flexibility of the procedure is also beneficial to endoscopists who choose the procedure to perform depending on the difficulty of the lesion, such as its location and association with colonic folds. In general, if the lesion's visibility is vertical, snare resection, such as hybrid ESD, is suitable because the cut line can be seen easily and the snare can be placed accurately in sight. However, if the lesion's visibility is tangential, conventional ESD is appropriate because submucosal dissection is easier in the tangential view than in the vertical view and snare placement is difficult in the tangential view.

Out of the 108 lesions analyzed in our study, 30 SSA/P were included. A recent development in molecular biology revealed a carcinogenic pathway-related SSA/P histology. ${ }^{17}$ However, it is controversial whether ESD can be applied in the treatment of large SSA/P. ${ }^{18}$ Our study showed that pre-cutting EMR and hybrid ESD for large SSA/P may potentially be feasible in daily clinical practice.

Simplification of ESD procedure through the development of modified methods is still required because of the gap in technical difficulties between conventional EMR and full ESD, especially for non-expert endoscopists. Precutting EMR and hybrid ESD are useful for beginners performing colorectal ESD as a bridge procedure between conventional EMR and conventional ESD..$^{19}$ However, by using multifunctional snare SOUTEN, the procedure time can be shorter not only because endoscopists can remove the lesion by easily through precutting EMR and hybrid ESD but also because SOUTEN can be used all through the procedure without changing the devices.

There are several limitations to our study. Firstly, we must acknowledge the general limitations of a retrospective single-center study. We did not have a control arm to compare the advantages and disadvantages of this new snare. Secondly, we did not apply consider time limit to snare the lesion; thus, the time for snaring and the choice of procedure to perform depended on the endoscopists. At the time of the study, we did not have sufficient time to perform a meaningful analysis of lesion recurrence. However, we expect a lower recurrence rate with our stepwise endoscopic protocol than with conventional EMR because our en block resection rate almost reached $100 \%$ in this study. Technically, the cutting line cannot be determined accurately with conventional EMR because of technical difficulties with placing the snare accurately; however, with pre-cutting EMR and hybrid ESD, endoscopists can determine the cutting line with an appropriate margin using cutting device, such as endoscopic knives. As already described, we used the multifunctional snare SOUTEN in determining the cutting line.

As there is growing economic concern for endoscopic procedures, SOUTEN has a great advantage because endoscopists can change the procedure accordingly without changing the equipment and incurring additional costs. By using SOUTEN, precutting EMR and hybrid ESD were successfully performed on tumors measuring $10-30 \mathrm{~mm}$ with a shorter procedure time and without major complication compared to conventional ESD.

\section{Conflicts of Interest}

The authors have no financial conflicts of interest.

\author{
Author Contributions \\ Conceptualization: Shinji Yoshii \\ Data curation: SY, Marina Kubo, Mio Matsumoto \\ Formal analysis: SY, Takefumi Kikuchi \\ Investigation: SY, MK \\ Methodology: SY \\ Project administration: SY \\ Resources: SY, MK, MM, Yasunari Takakuwa \\ Supervision: TK \\ Writing-original draft: SY \\ Writing-review\&editing: SY, TK
}

\section{Supplementary Material}

Video 1. Hybrid endoscopic submucosal dissection was performed to resect a 30-mm granular type laterally spreading tumor located in the lower rectum. En bloc resection was achieved within 9 minutes using SOUTEN without any adverse events (https://doi.org/10.5946/ce.2019.117.v001).

\section{ORCID}

Marina Kubo: https://orcid.org/0000-0001-9956-134X

Mio Matsumoto: https://orcid.org/0000-0002-3715-9598

Takefumi Kikuchi: https://orcid.org/0000-0003-4832-8681

Yasunari Takakuwa: https://orcid.org/0000-0003-4997-8969

\section{REFERENCES}

1. Tanaka S, Kashida H, Saito Y, et al. JGES guidelines for colorectal endoscopic submucosal dissection/endoscopic mucosal resection. Dig Endosc 2015;27:417-434.

2. Pohl H, Srivastava A, Bensen SP, et al. Incomplete polyp resection during colonoscopy-results of the complete adenoma resection (CARE) study. Gastroenterology 2013;144:74-80.el.

3. Saito Y, Fukuzawa M, Matsuda T, et al. Clinical outcome of endoscopic 
submucosal dissection versus endoscopic mucosal resection of large colorectal tumors as determined by curative resection. Surg Endosc 2010;24:343-352.

4. Terasaki M, Tanaka S, Oka S, et al. Clinical outcomes of endoscopic submucosal dissection and endoscopic mucosal resection for laterally spreading tumors larger than $20 \mathrm{~mm}$. J Gastroenterol Hepatol 2012;27:734-740.

5. Watanabe T, Muro K, Ajioka Y, et al. Japanese Society for Cancer of the Colon and Rectum (JSCCR) guidelines 2016 for the treatment of colorectal cancer. Int J Clin Oncol 2018;23:1-34.

6. Belderbos TD, Leenders M, Moons LM, Siersema PD. Local recurrence after endoscopic mucosal resection of nonpedunculated colorectal lesions: systematic review and meta-analysis. Endoscopy 2014;46:388-402.

7. Sakamoto T, Matsuda T, Nakajima T, Saito Y. Efficacy of endoscopic mucosal resection with circumferential incision for patients with large colorectal tumors. Clin Gastroenterol Hepatol 2012;10:22-26.

8. Lee EJ, Lee JB, Lee SH, Youk EG. Endoscopic treatment of large colorectal tumors: comparison of endoscopic mucosal resection, endoscopic mucosal resection-precutting, and endoscopic submucosal dissection. Surg Endosc 2012;26:2220-2230.

9. Yang DH, Kwak MS, Park SH, et al. Endoscopic mucosal resection with circumferential mucosal incision for colorectal neoplasms: comparison with endoscopic submucosal dissection and between two endoscopists with different experiences. Clin Endosc 2017;50:379-387.

10. Ohata K, Muramoto T, Minato Y, Chiba H, Sakai E, Matsuhashi N. Usefulness of a multifunctional snare designed for colorectal hybrid endoscopic submucosal dissection (with video). Endosc Int Open 2018;6:E249-E253.

11. Kudo S. Endoscopic mucosal resection of flat and depressed types of early colorectal cancer. Endoscopy 1993;25:455-461.

12. Minamino H, Nagami Y, Shiba M, et al. Colorectal polyps located across a fold are difficult to resect completely using endoscopic mucosal resection: a propensity score analysis. United European Gastroenterol J 2018;6:1547-1555.

13. Schlemper RJ, Riddell RH, Kato Y, et al. The Vienna classification of gastrointestinal epithelial neoplasia. Gut 2000;47:251-255.

14. Kanda Y. Investigation of the freely available easy-to-use software 'EZR' for medical statistics. Bone Marrow Transplant 2013;48:452-458.

15. Bae JH, Yang DH, Lee S, et al. Optimized hybrid endoscopic submucosal dissection for colorectal tumors: a randomized controlled trial. Gastrointest Endosc 2016;83:584-592.

16. Kim YJ, Kim ES, Cho KB, et al. Comparison of clinical outcomes among different endoscopic resection methods for treating colorectal neoplasia. Dig Dis Sci 2013;58:1727-1736.

17. Leggett $B$, Whitehall V. Role of the serrated pathway in colorectal cancer pathogenesis. Gastroenterology 2010;138:2088-2100.

18. Okamoto K, Muguruma N, Kagemoto K, et al. Efficacy of hybrid endoscopic submucosal dissection (ESD) as a rescue treatment in difficult colorectal ESD cases. Dig Endosc 2017;29(Suppl 2):45-52.

19. Toyonaga T, Man-I M, Morita Y, Azuma T. Endoscopic submucosal dissection (ESD) versus simplified/hybrid ESD. Gastrointest Endosc Clin N Am 2014;24:191-199. 